\title{
Long Noncoding RNA EGFR-AS1 Promotes Cell Proliferation by Increasing EGFR mRNA Stability in Gastric Cancer
}

\author{
Jiaojiao Hu Yingying Qian $^{\mathrm{d}} \quad$ Lipan Peng $^{\mathrm{e}} \quad$ Ling Ma ${ }^{\mathrm{a}} \quad$ Tianzhu Qiu ${ }^{\mathrm{a}}$ Yiqian Liu \\ Xiao Li ${ }^{\mathrm{b}} \quad$ Xiaofeng Chen ${ }^{\mathrm{a}}$ \\ aDepartment of Oncology, the First Affiliated Hospital of Nanjing Medical University, Nanjing \\ bDepartment of Pathology, the First Affiliated Hospital of Nanjing Medical University, Nanjing 'Nanjing \\ Maternity and Child Health Care Institute, Nanjing Maternity and Child Health Care Hospital, Obsterics \\ and Gynecology Hospital Affiliated to Nanjing Medical University, Nanjing ${ }^{\mathrm{d} D e p a r t m e n t}$ of Respiratory \\ Medicine, Nanjing First Hospital, Nanjing Medical University, Nanjing eDepartment of Gastrointestinal \\ Surgery, Shandong Provincial Hospital Affiliated to Shandong University, Jinan (China)
}

\section{Key Words}

IncRNAs • Gastric cancer • EGFR-AS1 • EGFR • MRNA stability • PI3K

\begin{abstract}
Background/Aims: LncRNA EGFR-AS1 is an antisense transcript of EGFR, which plays a key role in gastric cancer progression. This study was aimed to explore the effects of IncRNA EGFR-AS1 on GC and the underling mechanisms. Methods: The silencing of EGFR-AS1 expression was performed by using EGFR-AS1 shRNA lentivirus in MGC803 and SGC-7901 GC cell. The levels of IncRNA EGFR-AS1 and EGFR were detected by qPCR and western blot. Cell proliferation was assessed by CCK-8, EdU, and colony formation assays. The EGFR mRNA stability was explored by using RNA synthesis inhibitor $\alpha$-amanitin. Results: In our study, EGFR-AS1 significantly up-regulated in GC tissues and correlated with tumor size. And the expression of EGFR-AS1 positively correlated with EGFR in tissues. Moreover, knock-down of EGFR-AS1 inhibited the proliferation of GC cells via suppressing EGFR-dependent PI3K/AKT pathway in vitro and in vivo. Mechanismly, depletion of EGFR-AS1 was found to decrease EGFR expression by reduction of EGFR mRNA stability. Conclusion: Our findings suggested that EGFR-AS1 might have an oncogenic effect on GC and serve as a potential target of GC.
\end{abstract}

\section{Introduction}

Gastric cancer (GC) is one of the most common malignancies and the second leading cause of cancer-related death worldwide [1,2]. Despite the diagnostic and treatment have

J. Hu, Y. Qian and L. Peng contributed equally to this work.

\begin{tabular}{ll}
\hline Xiaofeng Chen & Dept. of Oncology, the First Affiliated Hospital of Nanjing Med. University \\
and Xiao Li & No. 300 Guangzhou Road, Nanjing, Jiangsu Province, 210029 (China) \\
& Tel. +86-25-83714511, E-Mail chenxiaofengnjmu@163.com; xiaolinjmu@aliyun.com
\end{tabular}


developed quickly, the 5-year overall survival rate remains unsatisfactory [3]. Molecular targeted therapy could reduce side effects and provides a good new way for the treatment of GC [4-6]. Therefore, revealing the underlying molecular mechanisms that contribute to gastric carcinogenesis is essential for the development of novel effective therapies for GC patients.

Long noncoding RNAs (lncRNAs) is a class of functional RNAs that transcripts longer than 200 nucleotides and without protein coding potential $[7,8]$. In recent years, thousands of lncRNAs have been discovered and findings have suggested that lncRNAs have vital roles in GC [9-15]. However, the functions and mechanisms of lncRNAs responsible for the development and progression of GC still need to be understood.

Epidermal growth factor receptor (EGFR) belongs to the family of receptor tyrosine kinases ErbB [16]. EGFR plays an important role in cell migration, proliferation, cell cycle regulation and other physiological processes [7]. EGFR is up-regulated in various cancers including gastric cancer $[12,13]$, and is recognized as oncogenic driver in tumorigenesis. LncRNA EGFR antisense RNA 1 (EGFR-AS1) is a 2.8-kb transcript that transcribes in the antisense strand of EGFR $[17,18]$. Reports have shown that overexpression of EGFR-AS1 was sufficient to induce resistance to tyrosine kinase inhibitors and knockdown of EGFRAS1 induces sustained tumor regression of squamous cell carcinoma [17]. EGFR-AS1 was relatively up-regulated in hepatocellular carcinoma (HCC) tissues and was determined to improve the ability of invasion and proliferation of HCC cells in vitro and in vivo via increasing the expression of EGFR [18]. However, the biological functions of EGFR-AS1 in GC have not been reported.

In the present study, we focused on the investigation of the function of EGFR-AS1 in GC. We analyzed the expression level of EGFR-AS1 in tumor and adjacent tissues and found that EGFR-AS1 was significantly upregulated in GC tumor tissues. We demonstrated that EGFRAS1 up-regulates EGFR expression through enhancing EGFR mRNA stability, thus resulting in activating the EGFR-dependent pathway and promoting the proliferation of gastric carcinoma cells in vitro and in vivo.

\section{Materials and Methods}

Ethical conduct of research

All the patients provided their written informed consent in accordance with the Declaration of Helsinki before enrollment in the study. The study was approved by the Institutional Review Board of the Nanjing Medical University (Nanjing, China).

Patients and samples

A total of $58 \mathrm{GC}$ tissues and pair adjacent normal gastric tissues were obtained with informed consent from patients who underwent radical resection surgery at the First Affiliated Hospital of Nanjing Medical University. No patient had the history of exposure to either radiotherapy or chemotherapy before the surgery, and no other co-occurrence cancers was diagnosed. This study was performed with the approval of the Medical Ethics Committee of the Nanjing Medical University.

\section{Cell culture}

The human gastric epithelial cell line GES-1 and GC cell lines (SGC7901, BGC823, MGC803 and MKN28) were purchased from the Cell Bank of the Chinese Academy of Science (Shanghai, China). The cells were grown in RPMI Medium 1640 (Gibco, CA, USA) supplemented with 10\% fetal bovine serum (Gibco, CA, USA), $100 \mathrm{U} / \mathrm{ml}$ penicillin and $100 \mathrm{mg} / \mathrm{ml}$ streptomycin in a humidified incubator of $5 \% \mathrm{CO}_{2}$ and $95 \%$ air at $37^{\circ} \mathrm{C}$.

RNA stability assay

For the analysis of RNA stability, GC cells were treated with $\alpha$-amanitin ( $50 \mu \mathrm{g} / \mathrm{ml}$, Sigma-Aldrich, USA). Cells were collected at different time points $(0,6,12,18$ and $24 \mathrm{~h})$ and total RNA were extracted using Trizol 


\section{Cellular Physiology Cell Physiol Biochem 2018;49:322-334 and Biochemistry \begin{tabular}{l|l} 
DOI: 10.1159/000492883 & (c) 2018 The Author(s). Published by S. Karger AG, Basel \\
www.karger.com/cpb
\end{tabular}

Hu et al.: Long Noncoding RNA EGFR-AS1 in Gastric Cancer

reagent (Invitrogen, USA). The level of mRNA was measured by qRT-PCR and normalized to 18S rRNA, the percentage of remaining RNA were relative to time 0 .

\section{Plasmid construction}

The DNA segments of EGFR-AS1-shRNA (shRNA-1 and shRNA-2) and scramble negative controls (shNC) were synthesized by GENEWIZ (Suzhou, China). The annealed duplex DNA was ligated into the 5'Xho I and 3'Not I sites of the lentiviral pLL3.7-GFP vector. The EGFR-AS1 shRNA sequences are listed in Table 1.

\section{Lentivirus Production and Infection}

Recombinant lentivirus was generated from 293T cells by co-transfection of pdelta-8.91 and pVSVG together with pLL3.7-shNC and pLL3.7-shRNA1/2 using Lipofectamine 2000 (Invitrogen, CA, USA). Lentivirus particles were harvested at 48 and 72 hours after transfection, and filtered through a $0.45 \mu \mathrm{m}$ filter, followed by concentrating 100-fold via ultracentrifugation. The virus-containing pellet was resuspended in DMEM, then aliquoted and stored at $-80^{\circ} \mathrm{C}$. Lentivirus particles were added to the GC cells in the presence of polybrene (Sigma, St. Louis, MO, USA). The supernatant was replaced with complete culture media after 24 hours. The interference efficiency of EGFR-AS1 in the infected cells was confirmed by qRTPCR at 48 hours after infection.

\section{Cell proliferation assays}

The cells were seeded in 96-well plates and measured using Cell Counting Kit-8 (Dojindo Laboratories, Kumamoto, Japan). Cell proliferation was determined every $24 \mathrm{~h}$ for 4 days. The optical density was measured with a microplate reader (Bio-Rad, Hercules, CA, USA). For the colony formation assay, $1.5 \times 10^{3}$ cells were seeded in a six-well plate. After 14 days, colonies were fixed and stained with $0.05 \%$ crystal violate (Invitrogen, Carlsbad, CA, USA) before counting. EdU immunofluorescence staining were performed

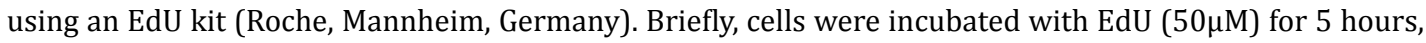
fixed with $4 \%$ paraformaldehyde and followed with permeabilization using PBS containing 0.5\% Triton $\mathrm{X}-100$. Then the cells were incubated in Apollo staining solution, then repeated permeation and wash, and incubated in Hoechst 33342. All experiments were performed in triplicate.

RNA extraction and quantitative RT-PCR ( $q R T-P C R$ )

Total RNA was extracted from cells using a TRIzol reagent kit (Invitrogen, CA, USA). Subsequently, RNA was reverse transcribed to cDNA from $1 \mu \mathrm{g}$ of total RNA using Oligo(dT) and random 6 mers oligos for priming. The qRT-PCR was carried out using the SYBR Premix Ex Taq in the StepOne Plus system (Applied Biosystems, Foster City, CA). The expression level of each gene was normalized by 18S rRNA and reported

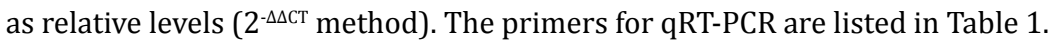

Table 1. Primers and RNA sequences used in this study

\begin{tabular}{lc}
\hline $\begin{array}{l}\text { oligonucleotide } \\
\text { sequences }\end{array}$ & Sequence $\left(5^{\prime}\right.$ to $3^{\prime}$ ) \\
\hline primers for Real-time PCR & GGCCATCACGTAGGCTTCCT \\
EGFR-AS1-qF & TGCGTCTTCACCTGGAAGGG \\
EGFR-AS1-qR & CCAAGGCACGAGTAACAAGCT \\
EGFR-qF & GCACATAGGTAATTTCCAAA \\
EGFR-qR & TTAATTCCGATAACGAACGAGA \\
18s rRNA-qF & CGCTGAGCCAGTCAGTGTAG \\
18s rRNA-qR & CGCTTCGGCAGCACATATACTA \\
U6-qF & CGCTTCACGAATTTGCGTGTCA \\
U6-qR & ACAACTTTGGTATCGTGGAAGG \\
$\begin{array}{l}\text { GAPDH-qF } \\
\text { GAPDH-qR }\end{array}$ & GCCATCACGCCACAGTTTC \\
Oligos for plasmid construction & TCGAGCGCAGCATCTCCTGATTACCTATTCAAGAGATAGGTAATCAGGAGATGCTGTTT \\
EGFR-AS1-sh1 & TTTTC \\
EGFR-AS1-sh2 & TCGAGCGCAGGCACTGATTTGTGCACAATCAAGAGTTGTGCACAAATCAGTGCCTGTTT \\
EGFR-AS1-shNC & TTTTGC \\
\hline
\end{tabular}




\section{Cellular Physiology Cell Physiol Biochem 2018;49:322-334 \begin{tabular}{ll|l} 
and Biochemistry Published onlIne: 23 August, 2018 & $\begin{array}{l}\text { (c) } 2018 \text { The Author(s). Published by S. Karger AG, Basel } \\
\text { www.karger.com/cpb }\end{array}$
\end{tabular}}

Hu et al.: Long Noncoding RNA EGFR-AS1 in Gastric Cancer

Western blot analysis

The proteins were extracted as previously described [19]. Equal amounts of protein (30 $\mu$ g) were loaded into and separated by $10 \%$ or $12 \%$ SDS-PAGE and transferred onto nitrocellulose membranes (Millipore, MA, USA). Then incubated at room temperature in block buffer BSA (5\%w/v in PBS) and followed by incubating with specific antibodies. Primary antibodies against GAPDH (Bioworld Technology), EGFR, p-EGFR, PI3K and p-PI3K (Cell Signaling Technology) were incubated overnight at $4^{\circ} \mathrm{C}$ The signal was detected with horseradish peroxidase-conjugated secondary antibodies and revealed using an ECL kit (Thermo Scientific). GAPDH protein was used as an internal control. Each experiment was repeated at least 3 times.

\section{Subcellular fractionation location}

RNA of nuclear and cytosolic fractions was separated using the PARIS Kit (Life Technologies, Carlsbad, CA, USA) as previously described [19]. The expression of U6, GAPDH and EGFR-AS1 were detected by qRTPCR. The primers for qRT-PCR are listed in Table 1.

\section{Xenograft HCC mice model}

Approximately $1 \times 10^{6}$ cells stably infected with EGFR-AS1 shRNA were harvested and injected subcutaneously into 4-week-old BALB/C nude mice. Tumor growth were measured every week from injection and calculated with the formula (length $\times$ width $^{2} \times 0.5$ ). After 5 weeks, mice were sacrificed, and the tumors were excised and measured. All animal experiments were conducted in accordance with the institutional standard guidelines of Nanjing Medical University and all experimental protocols were approved by the Use Committee for Animal Care of Jiangsu Province.

\section{Statistical analysis}

Statistical analyses were performed using SPSS 20.0 and GraphPad Prism 7. Expression of EGFR-AS1 in samples between GC tissues and adjacent gastric tissues was compared by a Wilcoxon signed-rank test. Correlations between the EGFR-AS1 expression levels and pathological features were analyzed with the chi square $(\chi 2)$ test. The Student t-test or one-way ANOVA was used to compare the results and expressed as mean \pm s.d. between any two preselected groups. Pearson's correlation coefficient was calculated using Prism7 software (GraphPad). A $P$ value $<0.05$ was considered statistically significant.

\section{Results}

The antisense transcribed IncRNA EGFRAS1 upregulated in GC tissues and cell lines

Firstly, we tested EGFR-AS1 expression in GC tissues and pair-matched adjacent gastric tissues from 58 GC patients by quantitative RTPCR (qRT-PCR). As shown in Fig. 1A, EGFR-AS1 expression was significantly increased in GC tissues compared with adjacent gastric tissues

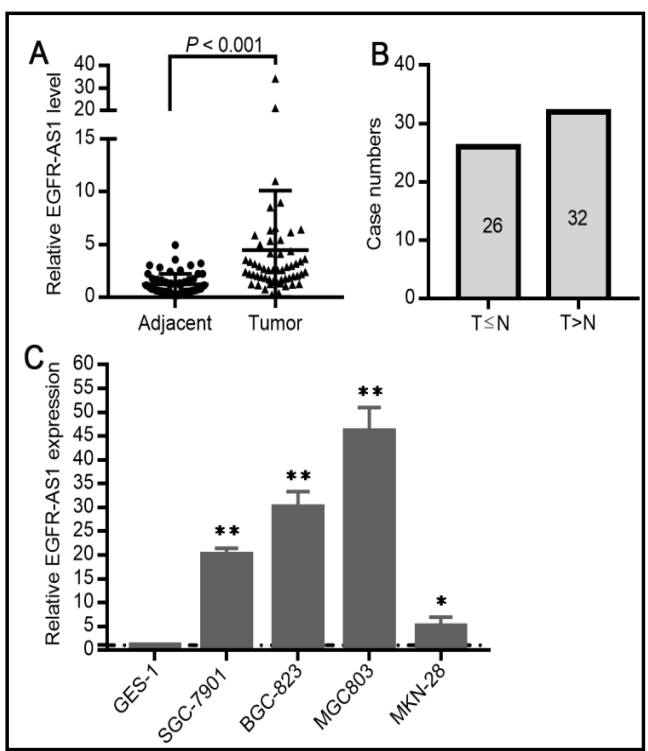

Fig. 1. EGFR-AS1 is highly expressed in GC specimens and cell lines. (A) The expression of EGFR-AS1 in 58 paired GC and adjacent gastric tissues were detected by qRT-PCR, the $18 \mathrm{~S}$ rRNA served as internal control. Significant differences were analyzed according to the Wilcoxon signedrank test $(\mathrm{P}<0.05$ are considered as significant). (B) The expression of EGFR-AS1 was at higher level in 55\% tumor tissues as compared to adjacent gastric tissues (32/58). $\mathrm{T} \leq \mathrm{N}$ : The expression of EGFR-AS1 in GC tissues was less than or equal to that in paired adjacent gastric tissues. $\mathrm{T}>\mathrm{N}$ : The expression of EGFR-AS1 in GC tissues was more than that in paired adjacent gastric tissues. (C) The levels of EGFR-AS1 were detected in GES-1 and four human GC cell lines by qRT-PCR. The values indicate the mean \pm s.d. for three separate experiments $\left({ }^{*} \mathrm{P}<0.05\right.$, ** $\mathrm{P}<0.01$, ANOVA). 
(Wilcoxon signed-rank test, $P<0.001)$. Compared to corresponding adjacent gastric tissues, the expression level of EGFR-AS1 was upregulated in 55\% tumor tissues (32/58) (Fig. 1B).

To investigate the correlation between EGFR-AS1 expression and patient clinical features, patients of GC were divided into EGFR-AS1 high and low expression groups depending on whether EGFR-AS1 expression was upregulated compared with the corresponding adjacent gastric tissues.) (Table 2). Here we found that higher EGFR-AS1 expression levels (67.6\%) were significantly correlated with larger tumor size $(P=0.023)$. However, there was no significant correlation between EGFR-AS1 expression and other clinicopathological features.

We further examined the expression level of EGFR-AS1 in normal gastric epithelium cell line GES1 and GC cell lines (SGC7901, BGC823, MGC803 and MKN-28). EGFR-AS1 expression in GC cell lines were significantly higher than in normal gastric epithelium cell line GES1 (Fig. 1C). All of the results above indicated a potential vital oncogenic role of EGFR-AS1 in GC.

\section{Knockdown of EGFR-AS1 suppressed GC cell proliferation in vitro}

As EGFR-AS1 was significantly upregulated in GC and associated with tumor size of patients, we next explored whether EGFR-AS1 plays a vital role in the cell proliferation of GC. MGC803 and SGC-7901 cell lines were selected for further study. We silenced the expression of EGFR-AS1 by using EGFR-AS1 shRNA lentivirus (two specific shRNAs target EGFR-AS1), and the interference efficiency was evaluated by qRT-PCR (Fig. 2A). Then, shRNA-2 was selected for following experiments due to its higher interference efficiency. CCK8 assay showed that cells displayed a markedly reduced proliferation rate with knockdown of EGFRAS1 in both of MGC803 and SGC-7901 cells (Fig. 2B). EdU staining assay demonstrated that decreased EGFR-AS1 contributed to less EdU-positive cells than control (Fig. 2C). To further confirm our observation, colony-formation assay was performed. Findings showed that silenced EGFR-AS1 expression significantly inhibited the clonogenicity of the MGC803 and SGC-7901 cells (Fig. 2D). These results suggest that EGFR-AS1 plays an important role in regulating GC cell proliferation.

\section{EGFR was positively correlated with the level of EGFR-AS1 in GC tissues}

EGFR-AS1 is transcribed in the opposite strand of EGFR. The genomic location region of EGFR-AS1 and EGFR is shown in Fig. 3A. The RNA-seq projects of PRJNA280600 and PRJEB4337 which are provided by NCBI database (Fig. 3B and 3C) and the GTEX RNA-seq

Table 2. Correlation between EGFR-AS1 expression and clinicopathological characteristics of gastric cancer patients $(n=58)$ \#EGFR-AS1 levels in tumor tissues were categorized as low or high depending on whether EGFR-AS1 expression was upregulated compared with the corresponding adjacent gastric tissues. TNM, Tumor node metastasis; *, P $<0.05$

\begin{tabular}{|c|c|c|c|c|c|}
\hline \multicolumn{6}{|c|}{ EGFR-AS1 expression\# } \\
\hline Characteristics & Low & $(n=26)$ & High $(n=32)$ & Chi-square & $\mathrm{P}$ value \\
\hline Age & & & & 0.120 & 0.655 \\
\hline$\leq 60$ & & 19 & 25 & & \\
\hline$>60$ & & 7 & 7 & & \\
\hline Gender & & & & 0.104 & 0.747 \\
\hline Male & & 16 & 21 & & \\
\hline Female & & 10 & 11 & & \\
\hline Differentiation grade & & & & 0.586 & 0.444 \\
\hline Well and Moderate & & 14 & 14 & & \\
\hline Poorly & & 12 & 18 & & \\
\hline Tumor size & & & & 5.17 & $0.023^{*}$ \\
\hline$<5 \mathrm{~cm}$ & & 15 & 9 & & \\
\hline$>5 \mathrm{~cm}$ & & 11 & 23 & & \\
\hline TNM stages & & & & 0.330 & 0.567 \\
\hline I-II & & 16 & 22 & & \\
\hline III-IV & & 10 & 10 & & \\
\hline Metastasis & & & & 0.22 & 0.639 \\
\hline Yes & & 17 & 19 & & \\
\hline No & & 9 & 13 & & \\
\hline
\end{tabular}




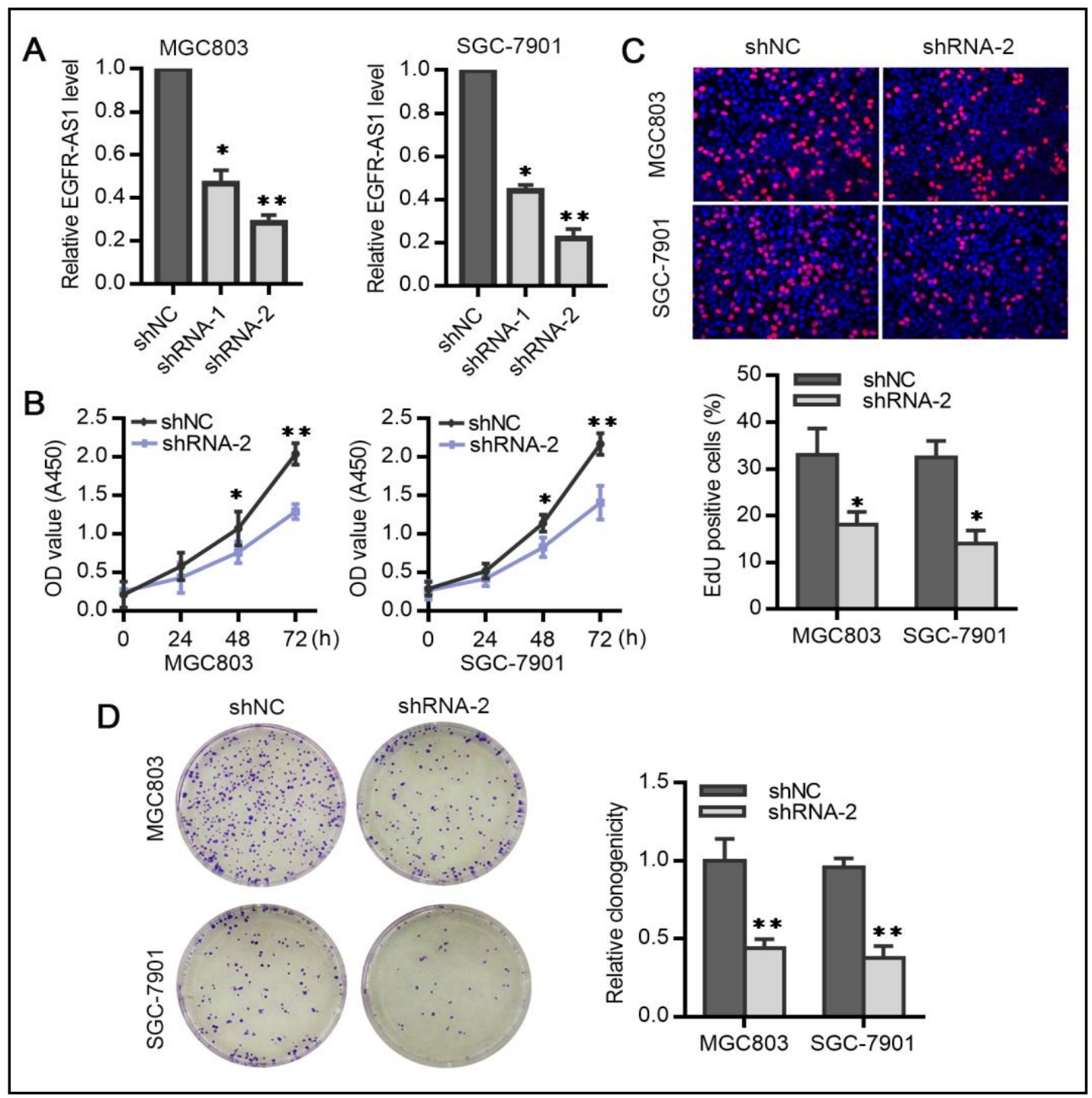

Fig. 2. EGFR-AS1 affect gastric carcinoma cell growth.(A) Expression level of EGFR-AS1 in MGC803 and SGC-7901 cells after infected with shNC and shEGFR-AS1-expressing lentivirus (represented by shNC or shRNA1/2). The values indicate the mean \pm s.d. for three separate experiments $\left({ }^{*} \mathrm{P}<0.05,{ }^{* *} \mathrm{P}<0.01, \mathrm{ANOVA}\right)$. (B) CCK-8 assay was detected in different time points including 0, 24, 48 and $72 \mathrm{~h}$ showed a decreased level of EGFR-AS1 inhibited the growth in MGC803 and SGC-7901 cell lines. The values indicate the mean \pm s.d. for three separate experiments $\left({ }^{*} \mathrm{P}<0.05,{ }^{* *} \mathrm{P}<0.01\right.$, ANOVA). (C) EdU staining analysis of cell proliferation. The blue color represents the nuclei, and the red color indicates EdU-positive nuclei (upper). The graphs show the percentage of EdU-positive nuclei (lower). The values indicate the mean \pm s.d. for three separate experiments $\left({ }^{*} \mathrm{P}<0.05\right.$, Student t-test). (D) Colony-forming assays were used to determine the colony ability of shRNA-2-infected MGC803 and SGC-7901 cells. The colonies were counted and are depicted in a bar chart (right panel). The values indicate the mean \pm s.d. for three separate experiments $\left({ }^{* *} \mathrm{P}<0.01\right.$, Student $\mathrm{t}$-test).

projects provided by UCSC database (Fig. 3D) show a similar expression pattern between EGFR and EGFR-AS1, especially with a very high level in placenta compared to other normal tissues (Fig. 3B and 3C). The database analysis suggests that EGFR-AS1 may have positive correlation with EGFR. Thus, we evaluated the correlation between EGFR-AS1 and EGFR. The mRNA levels of EGFR in 58 paired tumors and adjacent nontumor tissues were quantified by qRT-PCR, and we found a significantly increased level of EGFR in tumor group (Fig. 4A). 


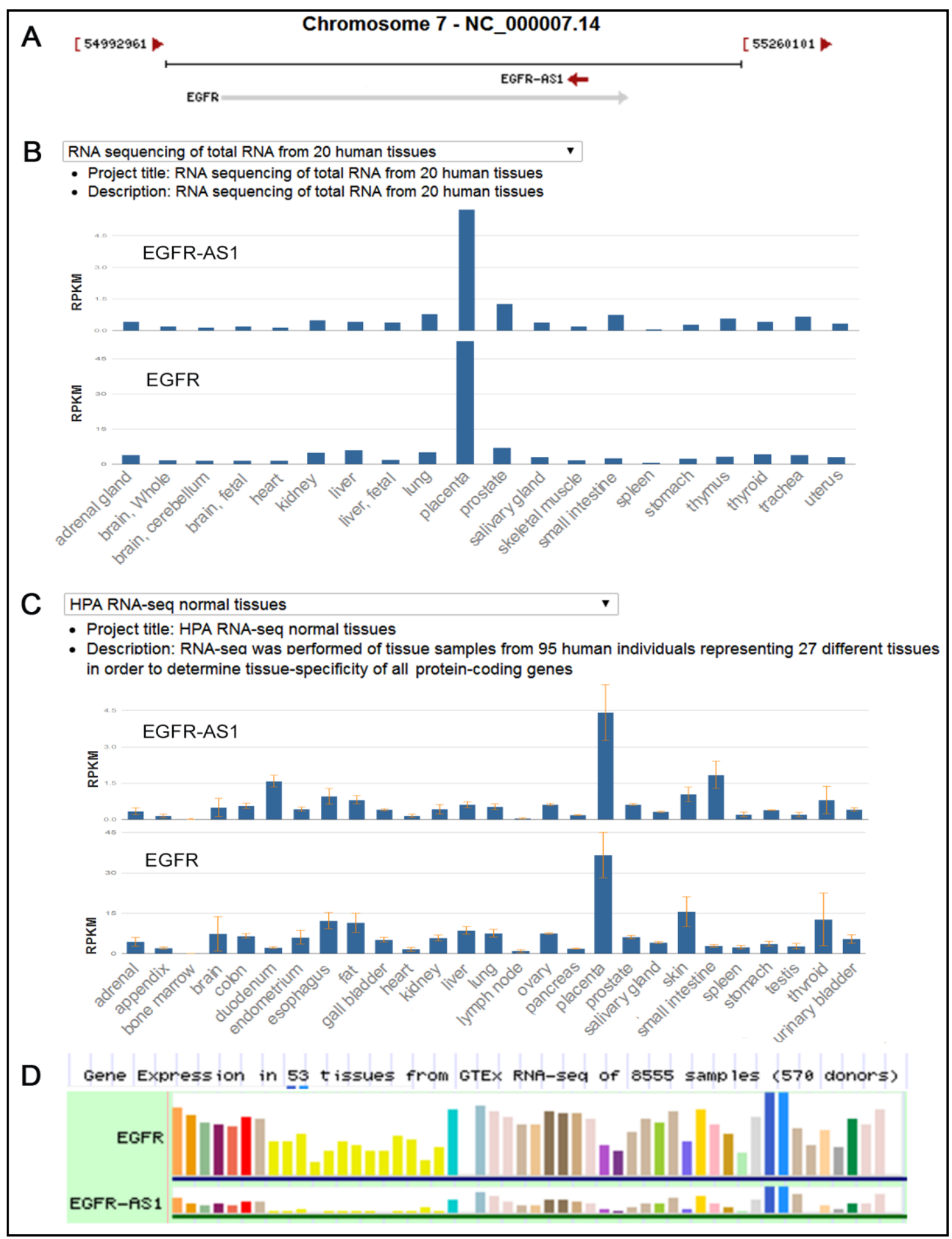

Fig. 3. Positive correlation between the level of EGFR and EGFR-AS1 in GC tissues.(A) Showing the genomic region of EGFR and EGFR-AS1 in NCBI database; arrows show the direction of transcription. Human EGFRAS1 is transcribed on the opposite strand. (B) The relative expression level of EGFR and EGFR-AS1 by RNA sequencing of total RNA from 20 human tissues in BioProject: PRJNA280600, and provided by NCBI database. (C) The relative expression level of EGFR and EGFR-AS1 by HPA RNA-seq of 27 different normal tissue samples from 95 human individuals in BioProject: PRJEB4337, and provided by NCBI database. (D) The relative expression level of EGFR and EGFR-AS1 in 53 tissues from GTEX RNA-seq of 8555 samples of 570 donors, and provided by UCSC database.

\section{KARGER}


Fig. 4. positive correlation between the level of EGFR and EGFR-AS1 in GC tissues.(A) qRT-PCR analysis of EGFR expression in 58 paired GC and adjacent gastric tissues, the $18 \mathrm{~S}$ rRNA served as internal control. Significant differences were analyzed according to the Wilcoxon signed-rank test $(\mathrm{P}<0.05$ are considered as significant, Student t-test). (B) The correlation between EGFR mRNA level and EGFR-AS1 level was be analyzed in tissues. The $\triangle \mathrm{Ct}$ values (normalized to $18 \mathrm{~S}$ rRNA) were subjected to Pearson correlation analysis. (C) Patients with GC were divided into EGFR-AS1 high/lowexpression groups based on whether EGFR-AS1 expression was upregulated compared with the corresponding adjacent gastric tissues. The expression of EGFR protein was compared in the
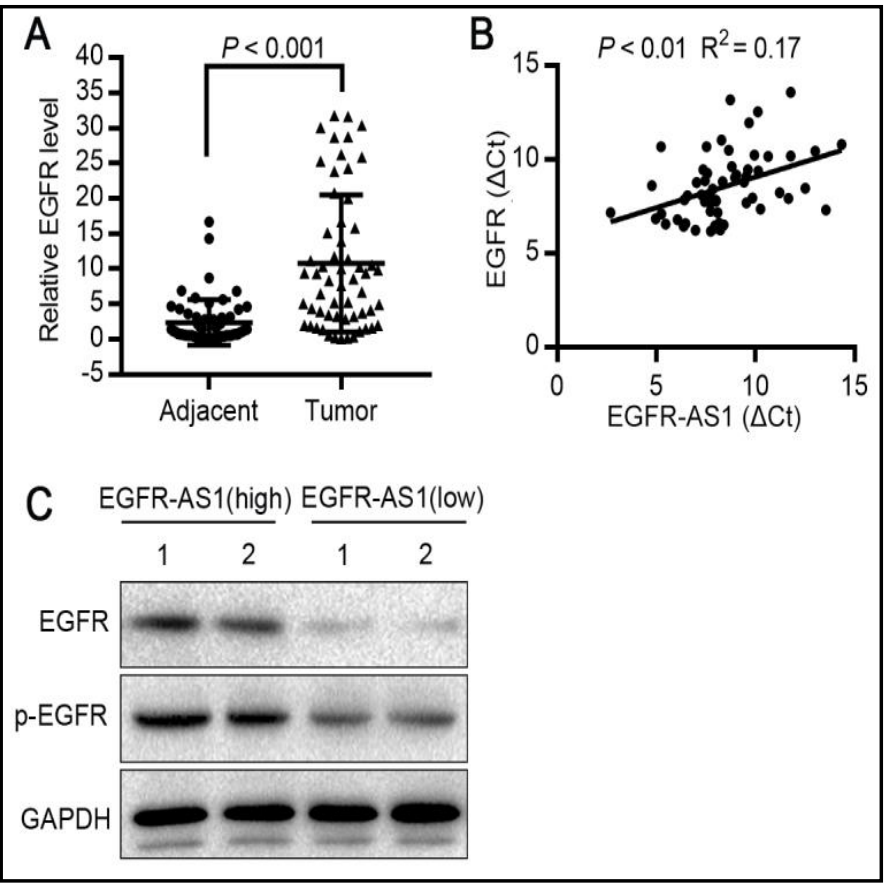
two groups by western blot assay. GAPDH served as the internal control.

A significantly positive correlation was also observed between the expression of EGFR and EGFR-AS1 $\left(\mathrm{R}^{2}=0.17, P<0.01\right.$, Pearson correlation analysis, Fig. 4B). Furthermore, the protein level of EGFR was also higher in EGFR-high samples compared to EGFR-low samples of the GC tissues (Fig. 4C).

Knockdown of EGFR-AS1 inhibited EGFR expression via reducing EGFR mRNA stability

We observed above that the expression of EGFR was positively correlated with EGFRAS1. Thus, we investigated whether EGFR-AS1 could influence the expression of EGFR. The knockdown efficiency of EGFR-AS1 in MGC803 and SGC-7901 cells were detected (Fig. $5 A)$. The mRNA and protein expression of EGFR were dramatically decreased in EGFR-AS1 knockdown cells (Fig. 5B and 5C). EGFR-dependent PI3K/AKT pathway plays a critical role in cell proliferation [20-22]. Consequently, we aim to investigate whether EGFR-AS1 promoted GC cell proliferation through activation of EGFR-dependent PI3K/AKT pathway. Results showed that EGFR-AS1 knockdown resulted in inhibition of the phosphorylation of EGFR and PI3K (p-EGFR and p-PI3K) in MGC803 cells treated with shRNA. (Fig. 5C).

We further identified the underlying mechanism for the upregulated expression of EGFR by EGFR-AS1. Because the EGFR-AS1 is transcribed on the opposite strand of EGFR, we analyzed the RNA sequence base pairing between EGFR-AS1 and EGFR mRNA. As shown in Fig. 5D, a long base-pairing with a length of 194bp has been predicted by NCBI BLAST (http://blast.ncbi.nlm.nih.gov/). It has been reported that a long base-pairing (104bp) of BACE1-AS and BACE1 mRNA promoted the mRNA stability of BACE1. Therefore, we tested the effect of EGFR-AS1 on EGFR mRNA stability. Cells were treated with RNA synthesis inhibitor $\alpha$-amanitin or DMSO (negative control), then the level of EGFR mRNA was measured by qRT-PCR and the percentage of remaining mRNA were relative to time 0 . Results showed a decreased stability of EGFR mRNA in EGFR-AS1 silenced MGC803 cells, however the knockdown of EGFR-AS1 did not altered the GAPDH (negative control) mRNA stability (Fig. 5E). Collectively, our data demonstrated that knockdown of EGFR-AS1 decreased EGFR expression by reducing EGFR mRNA stability. Finally, we also showed that EGFR-AS1 localized both in the cytosol and nucleus in MGC803 cells (Fig. 5F), indicating a wide range of regulatory mechanism of EGFR-AS1 for target gene. 


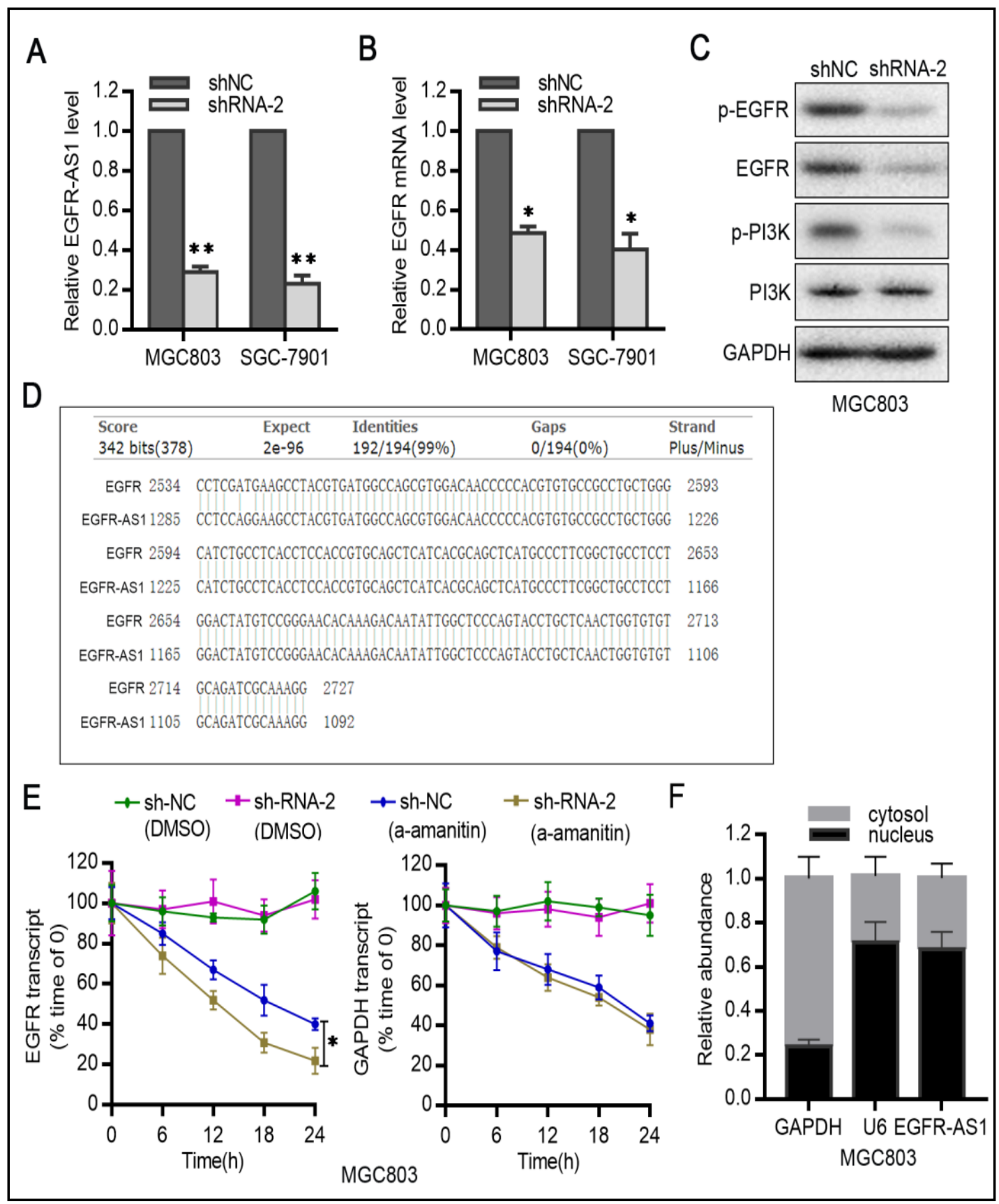

Fig. 5. knockdown of EGFR-AS1 decrease EGFR expression by reducing EGFR mRNA stability. (A) EGFRAS1 expression levels after infection of EGFR-AS1 shRNA or control in MGC803 and SGC-7901 cells. The values indicate the mean \pm s.d. for three separate experiments $\left({ }^{*} \mathrm{P}<0.05,{ }^{* *} \mathrm{P}<0.01\right.$, Student $\mathrm{t}$-test). (B) The relative levels of EGFR mRNA were examined by qRT-PCR, knockdown of EGFR-AS1 decrease EGFR mRNA expression. (C) Western blot assay showing the protein level of EGFR, p-EGFR, PI3K and p-PI3K upon silencing of EGFR-AS1 in MGC803. GAPDH served as the internal control. (D) The sequence presented here were the overlap region of EGFR-AS1 with EGFR mRNA (194 bp). (E) The mRNA stability of EGFR and GAPDH were tested by qRT-PCR every 6 h relative to time 0 after using $\alpha$-amanitin $(50 \mu \mathrm{M})(\mathrm{RNA}$ synthesis inhibitor) or dimethylsulfoxide (DMSO, negative control) in MGC803 cells, and normalized to 18S rRNA. The values indicate the mean \pm s.d. for three separate experiments $\left({ }^{*} \mathrm{P}<0.05\right.$, ANOVA). (F) GAPDH, U6 and EGFRAS1 RNA expression were detected in separated cytoplasm and nuclear RNA in MGC803 cells by qRT-PCR. GAPDH was used as the control for cytoplasmic expression and U6 for nuclear expression.

\section{KARGER}


Fig. 6. Inhibition of tumor growth by knockdown of EGFR-AS1 in vivo.(A) Photograph of actual tumor after removal from mice at 5 weeks after injection of MGC803 cells infected with EGFR-AS1 shNC and shRNA-2. (B) The volume of the subcutaneous tumors were measured after injection every week. The tumor growth curve shows that knockdown of EGFR-AS1 significantly inhibited tumor growth in the mice. Data are the mean

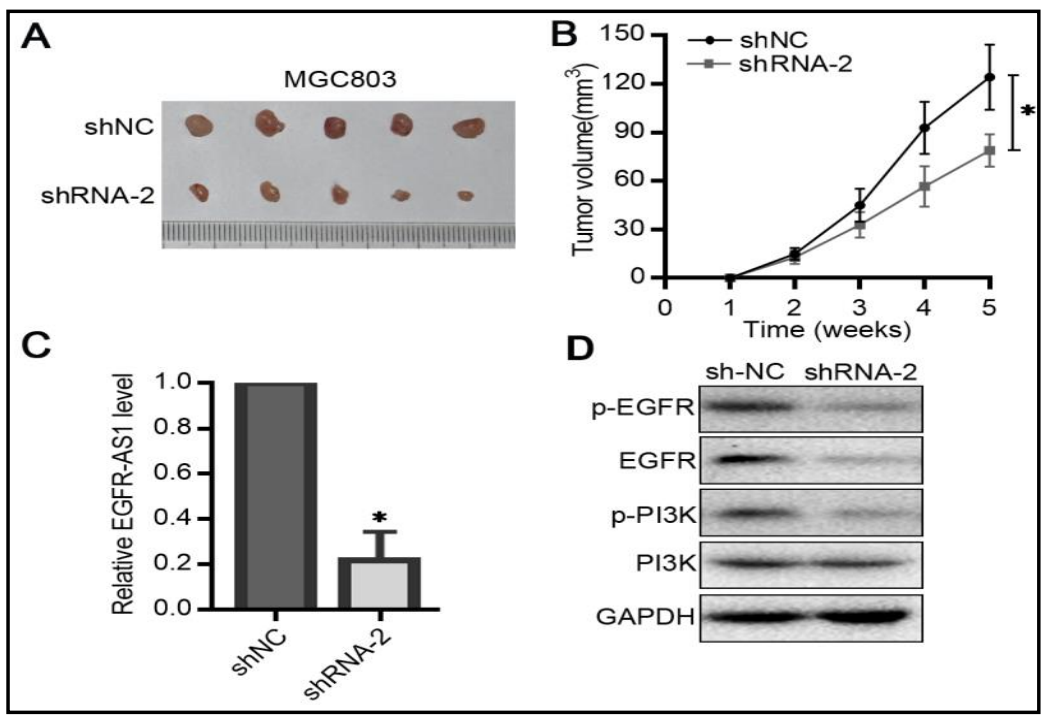
\pm s.d., Asterisk indicates a significant change $\left({ }^{*} \mathrm{P}<0.05,{ }^{* *} \mathrm{P}<0.01\right)$. (C) EGFR-AS1 expression levels were detected by qRT-PCR in tumors harvested form mice. $18 \mathrm{~S}$ rRNA was used as the control. The values indicate the mean \pm s.d. $\left({ }^{*} \mathrm{P}<0.05\right.$, Student t-test). (D) The protein level of EGFR, p-EGFR, PI3K and p-PI3K were detected by western blot in tumors harvested form mice. GAPDH served as the internal control.

EGFR-AS1 knockdown inhibited tumorigenesis of GC cells in vivo

We examined whether EGFR-AS1 influenced the growth of GC cells in nude mice in vivo. EGFR-AS1 silenced MGC803 cells (shRNA-2) or control cells (shNC) were subcutaneously injected into BALB/C nude mice, and tumor size was monitored every week. The results demonstrated that cells with EGFR-AS1 silenced MGC803 cells developed significantly smaller tumors than control cells (Fig. 6A), and as presented in Fig. 6B, knockdown of EGFRAS1 significantly suppressed the tumor growth curve compared with negative control group. Moreover, inhibition of EGFR-AS1, p-EGFR and p-PI3K was also observed in tumors of mice with EGFR-AS1 silenced MGC803 cells injection (Fig. 6C and 6D).

\section{Discussion}

LncRNAs have attracted great interest recent years due to its ability involved in multiple cellular processes, including proliferation, apoptosis, cell migration and invasion [23, 24]. LncRNAs display complicated regulatory function by a wide range of regulatory mechanism through transcriptional regulation, epigenetic modulation through chromatin modification and post-transcriptional regulation [25-30]. However, the involvement of IncRNAs in human GC development and progression are still need to be illustrated.

LncRNA EGFR-AS1 originates from the opposite strand of EGFR $[17,18]$. EGFR-AS1 has been reported relatively high expressed and up-regulated the expression of EGFR in HCC tissue, thus promoting HCC development by improving the ability of invasion and proliferation of HCC cells in vitro and in vivo [18]. EGFR-AS1 knockdown was sufficient to induce sustained tumor regression of squamous cell carcinoma in vivo by mediating EGFR addiction and activation of the EGFR pathway [17]. Thus, we attempted to investigate the role and molecular mechanism of EGFR-AS1 in gastric cancer.

We report here, for the first time, that EGFR-AS1 was expressed to a high degree in GC samples compared with adjacent gastric tissues. We observed that high expression level of EGFR-AS1 in GC patients was significantly correlated with tumor size, but no significant correlation between EGFR-AS1 expression and other clinicopathological features were 
found. Depletion of EGFR-AS1 expression inhibited GC cell proliferation in vitro and in vivo. These data suggest that EGFR-AS1 serves as an oncogenic lncRNA in gastric cancer. The expression of EGFR in clinical samples was found to be significantly higher in GC tissues than in adjacent non-tumor tissues, which is consistent with other reports [31]. Also, a significantly positive correlation was observed between the expression of EGFR and EGFRAS1. Additionally, decreased level of EGFR was found upon the knockdown of EGFR-AS1 expression. We also detected the activation of EGFR-dependent PI3K/AKT pathway, which played an important role in cell proliferation [20-22]. Our findings suggest that EGFR-AS1 induction of cell proliferation through activation of EGFR-dependent PI3K/AKT pathway in vitro and in vivo.

We further to identify the underlying molecular mechanism of EGFR-AS1 upregulated EGFR expression. LncRNAs have been reported to promote mRNA decay and repress the translation of mRNA by short partial base-pairing with specific target mRNAs [32,33]. On the contrary, longer base-pairing (104bp) of BACE1-AS (BACE1 antisense lncRNA) and BACE1 mRNA promoted the mRNA stability of BACE1 by protection of BACE1 mRNA protected from degradation [34]. A long base-pairing with a length of 194bp has been predicted between EGFR-AS1 and EGFR mRNA. Therefore, we investigated whether EGFR-AS1 promote the expression of EGFR through affecting mRNA stability. Results demonstrated that silence of EGFR-AS1 decreased EGFR mRNA stability. Collectively, our data demonstrate that knockdown of EGFR-AS1 decreases EGFR expression by reducing mRNA stability. What are the mechanisms about the upregulation of EGFR-AS1 in GC samples? The gene region with H3K4me1 and/or H3K27Ac modification of histone proteins is suggestive of enhancer and, to a lesser extent, other regulatory activity, which were observed on the EGFR-AS1 in 7 tumor cell lines by ChIP-seq results provided by UCSC database [35, 36]. Whether upregulation of EGFR-AS1 in GC due to the modification status of histone H3, and other mechanisms regarding EGFR-AS1 regulate EGFR expression are still need to be investigated in the future.

\section{Conclusion}

In conclusion, EGFR-AS1 expression were significantly increased in GC tissues and cell lines. Additionally, knockdown of EGFR-AS1 inhibited cell proliferation in vivo and in vitro through suppression of EGFR expression by reducing EGFR mRNA stability. Our findings suggest that high expression of EGFR-AS1 may have an oncogenic effect in GC, and may serve as potential targets for GC therapy in the future.

\section{Acknowledgements}

This work was supported by Jiangsu Provincial Natural Science Foundation-Youth Foundation (No. BK20170699) and Shandong Provincial Natural Science Foundation (No. ZR2015PH024). We thank the developers of 'SPSS' and the 'GraphPad Prism 7' for creating these useful platforms. X Li and XF Chen designed the study; X Li, J Hu, Y Qian, L Ma performed experiments and analyzed data; J Hu, X Li and X Chen analysis of data and drafting of the manuscript; T Qiu, Y Liu, L Peng and Y Liu collected clinical data and statistical analysis; All authors reviewed the manuscript before submission; All the authors approved the final version of the manuscript.

\section{Disclosure Statement}

The authors declare no conflicts of interest. 


\section{Cellular Physiology Cell Physiol Biochem 2018;49:322-334 \begin{tabular}{l|l} 
DOI: 10.1159/000492883 & Ond Biochemistry 2018 The Author(s). Published by S. Karger AG, Basel \\
wwww.karger.com/cpb
\end{tabular}

\section{References}

-1 Ferlay J, Soerjomataram I, Dikshit R, Eser S, Mathers C, Rebelo M, Parkin DM, Forman D, Bray F: Cancer incidence and mortality worldwide: sources, methods and major patterns in GLOBOCAN 2012. Int J Cancer 2015;136:359-386.

-2 Cancer Genome Atlas Research N: Comprehensive molecular characterization of gastric adenocarcinoma. Nature 2014;513:202-209.

3 Siegel R, Naishadham D, Jemal A: Cancer statistics, 2013. CA Cancer J Clin 2013;63:11-30.

-4 Van Cutsem E, Sagaert X, Topal B, Haustermans K, Prenen H: Gastric cancer. The Lancet 2016;388:26542664.

-5 Wang B, Yang H, Shen L, Wang J, Pu W, Chen Z, Shen X, Fu J, Zhuang Z: Rs56288038 (C/G) in 3'UTR of IRF-1 Regulated by MiR-502-5p Promotes Gastric Cancer Development. Cell Physiol Biochem 2016;40:391-399.

6 Wang X, Li T, Li M, Cao N, Han J: The Functional SOCS3 RS115785973 Variant Regulated by MiR-4308 Promotes Gastric Cancer Development in Chinese Population. Cell Physiol Biochem 2016;38:1796-1802.

7 Wilusz JE, Sunwoo H, Spector DL: Long noncoding RNAs: functional surprises from the RNA world. Genes Dev 2009;23:1494-1504.

8 Rinn JL, Chang HY: Genome regulation by long noncoding RNAs. Annu Rev Biochem 2012;81:145-166.

9 Liu Z, Chen Z, Fan R, Jiang B, Chen X, Chen Q, Nie F, Lu K, Sun M: Over-expressed long noncoding RNA HOXA11-AS promotes cell cycle progression and metastasis in gastric cancer. Mol Cancer 2017;16:82.

10 Sun M, Nie F, Wang Y, Zhang Z, Hou J, He D, Xie M, Xu L, De W, Wang Z, Wang J: LncRNA HOXA11-AS Promotes Proliferation and Invasion of Gastric Cancer by Scaffolding the Chromatin Modification Factors PRC2, LSD1, and DNMT1. Cancer Res 2016;76:6299-6310.

-11 Fang XY, Pan HF, Leng RX, Ye DQ: Long noncoding RNAs: novel insights into gastric cancer. Cancer Lett 2015;356:357-366.

12 Xu MD, Wang Y, Weng W, Wei P, Qi P, Zhang Q, Tan C, Ni SJ, Dong L, Yang Y, Lin W, Xu Q, Huang D, Huang Z, Ma Y, Zhang W, Sheng W, Du X: A Positive Feedback Loop of IncRNA-PVT1 and FOXM1 Facilitates Gastric Cancer Growth and Invasion. Clin Cancer Res 2017;23:2071-2080.

13 Li L, Geng Y, Feng R, Zhu Q, Miao B, Cao J, Fei S: The Human RNA Surveillance Factor UPF1 Modulates Gastric Cancer Progression by Targeting Long Non-Coding RNA MALAT1. Cell Physiol Biochem 2017;42:2194-2206.

14 Yan J, Zhang Y, She Q, Li X, Peng L, Wang X, Liu S, Shen X, Zhang W, Dong Y, Lu J, Zhang G: Long Noncoding RNA H19/miR-675 Axis Promotes Gastric Cancer via FADD/Caspase 8/Caspase 3 Signaling Pathway. Cell Physiol Biochem 2017;42:2364-2376.

15 Yan K, Tian J, Shi W, Xia H, Zhu Y: LncRNA SNHG6 is Associated with Poor Prognosis of Gastric Cancer and Promotes Cell Proliferation and EMT through Epigenetically Silencing p27 and Sponging miR-101-3p. Cell Physiol Biochem 2017;42:999-1012.

16 Patel R, Leung HY: Targeting the EGFR-family for therapy: biological challenges and clinical perspective. Curr Pharm Des 2012;18:2672-2679.

17 Tan DSW, Chong FT, Leong HS, Toh SY, Lau DP, Kwang XL, Zhang X, Sundaram GM, Tan GS, Chang MM, Chua BT, Lim WT, Tan EH, Ang MK, Lim TKH, Sampath P, Chowbay B, Skanderup AJ, DasGupta R, lyer NG: Long noncoding RNA EGFR-AS1 mediates epidermal growth factor receptor addiction and modulates treatment response in squamous cell carcinoma. Nat Med 2017;23:1167-1175.

-18 Qi HL, Li CS, Qian CW, Xiao YS, Yuan YF, Liu QY, Liu ZS: The long noncoding RNA, EGFR-AS1, a target of GHR, increases the expression of EGFR in hepatocellular carcinoma. Tumour Biol 2016;37:1079-1089.

19 Hu JJ, Song W, Zhang SD, Shen XH, Qiu XM, Wu HZ, Gong PH, Lu S, Zhao ZJ, He ML, Fan H: HBx-upregulated lncRNA UCA1 promotes cell growth and tumorigenesis by recruiting EZH2 and repressing p27Kip1/CDK2 signaling. Sci Rep 2016;6:23521.

20 Zheng X, Jiang F, Katakowski M, Zhang ZG, Lu QE, Chopp M: ADAM17 promotes breast cancer cell malignant phenotype through EGFR-PI3K-AKT activation. Cancer Biol Ther 2009;8:1045-1054. 


\section{Cellular Physiology Cell Physiol Biochem 2018;49:322-334 \begin{tabular}{l|l} 
DOI: 10.1159/000492883 & $\begin{array}{l}\text { O 2018 The Author(s). Published by S. Karger AG, Basel } \\
\text { www.karger.com/cpb }\end{array}$
\end{tabular}}

Hu et al.: Long Noncoding RNA EGFR-AS1 in Gastric Cancer

-21 Freudlsperger C, Burnett JR, Friedman JA, Kannabiran VR, Chen Z, Van Waes C: EGFR-PI3K-AKT-mTOR signaling in head and neck squamous cell carcinomas: attractive targets for molecular-oriented therapy. Expert Opin Ther Targets 2011;15:63-74.

22 Lin F, Lin P, Zhao D, Chen Y, Xiao L, Qin W, Li D, Chen H, Zhao B, Zou H, Zheng X, Yu X: Sox2 targets cyclinE, p27 and survivin to regulate androgen-independent human prostate cancer cell proliferation and apoptosis. Cell Prolif 2012;45:207-216.

-23 Xu D, Yang F, Yuan JH, Zhang L, Bi HS, Zhou CC, Liu F, Wang F, Sun SH: Long noncoding RNAs associated with liver regeneration 1 accelerates hepatocyte proliferation during liver regeneration by activating Wnt/betacatenin signaling. Hepatology 2013;58:739-751.

-24 Fatica A, Bozzoni I: Long non-coding RNAs: new players in cell differentiation and development. Nat Rev Genet 2014;15:7-21.

25 Huarte M: The emerging role of IncRNAs in cancer. Nat Med 2015;21:1253-1261.

26 Forrest ME, Khalil AM: Review: Regulation of the cancer epigenome by long non-coding RNAs. Cancer Lett 2017;407:106-112.

27 Wang KC, Chang HY: Molecular mechanisms of long noncoding RNAs. Mol Cell 2011;43:904-914.

28 Mercer TR, Mattick JS: Structure and function of long noncoding RNAs in epigenetic regulation. Nat Struct Mol Biol 2013;20:300-307.

29 Yoon JH, Abdelmohsen K, Gorospe M: Posttranscriptional gene regulation by long noncoding RNA. J Mol Biol 2013;425:3723-3730.

-30 Fatima F, Nawaz M: Vesiculated Long Non-Coding RNAs: Offshore Packages Deciphering Trans-Regulation between Cells, Cancer Progression and Resistance to Therapies. Noncoding RNA 2017;3:

31 Zhou J, Zhi X, Wang L, Wang W, Li Z, Tang J, Wang J, Zhang Q, Xu Z: Linc00152 promotes proliferation in gastric cancer through the EGFR-dependent pathway. J Exp Clin Cancer Res 2015;34:135.

32 Gong C, Maquat LE: IncRNAs transactivate STAU1-mediated mRNA decay by duplexing with 3' UTRs via Alu elements. Nature 2011;470:284-288.

-33 Wang H, Iacoangeli A, Lin D, Williams K, Denman RB, Hellen CU, Tiedge H: Dendritic BC1 RNA in translational control mechanisms. J Cell Biol 2005;171:811-821.

-34 Faghihi MA, Modarresi F, Khalil AM, Wood DE, Sahagan BG, Morgan TE, Finch CE, St Laurent G, 3rd, Kenny PJ, Wahlestedt C: Expression of a noncoding RNA is elevated in Alzheimer's disease and drives rapid feedforward regulation of beta-secretase. Nat Med 2008;14:723-730.

35 Hon GC, Hawkins RD, Ren B: Predictive chromatin signatures in the mammalian genome. Hum Mol Genet 2009;18:R195-201.

-36 Wang Z, Zang C, Rosenfeld JA, Schones DE, Barski A, Cuddapah S, Cui K, Roh TY, Peng W, Zhang MQ, Zhao $\mathrm{K}$ : Combinatorial patterns of histone acetylations and methylations in the human genome. Nat Genet 2008;40:897-903. 